\title{
NUEVAS EXPERIENCIAS EN URBANISMO. INTERNET AL SERVICIO DEL PLANEAMIENTO URBANO
}

\author{
(NEW EXPERIENCES ON PLANNING. INTERNET AT THE SERVICE OF CITY PLANNING)
}

\author{
Marina Álvarez-Alonso, L. Pérez Jiménez, J.A. Pérez Ruy-Díaz, N.A. Guevara Sanz, J.A. Hernández Sánchez \\ e I. Moreno Navarro
}

Facultad de Informática (U.P.M.). Boadilla del Monte-Madrid

ESPAÑA

Fecha de recepción: $27-$ VI-97

\section{RESUMEN}

Este articulo recoge la descripción de un trabajo piloto, consistente en la publicación del Avance de un Plan General de Urbanismo, en el cual se han utilizado las nuevas tecnologias que el mercado ofrece. Con este trabajo se pretende realizar un sistema de información de fácil acceso que proporcione una información eficaz a todos los ciudadanos y que, a su vez, facilite la gestión de las Instituciones Publicas.

El proyecto describe dos modos distintos de acceder a la información urbanistica. Una forma estática, modo habitual de representar la información en Internet, empleada por vez primera en el planeamiento urbanistico. Y una segunda forma, fundamentada en la conexión de un Sistema GIS a Internet, que permite acceder dinámicamente a la información almacenada en una base de datos.

Este trabajo se ha llevado a cabo en el Proyecto S.I.C. que, desarrollado en la Facultad de Informática de la Universidad Politécnica de Madrid, se enmarca dentro de la Acción Especial PASO del Ministerio de Industria y Energía y la Comunidad Europea.

\section{SUMMARY}

The present article is the description of a prototype. This job consists on the publication of a General City Planning Advance, where the newest technologies offered by the market have been used. Our intention is to perform an Information System, easy to access by everybody but powerful enough to offer a complete information to the citizens. We also are trying to provide the Public Institutions a fluent way to attend to the demands of the people.

The project describes two different ways of accessing the City Planning Information. The first manner is the classical way we can find in almost every website, though this is the first time that it is used for this purpose, the publication of a City Planning. The second one is based on the connection of a Geographical Information System through Internet, to provide dynamic access to the information stored in the database of the GIS.

This project has been developed trough the S.I.C. project, a project housed in the Facultad de Informatica of the Universidad Politécnica de Madrid and supported by the PASO Special Action from the Ministry of Industry and Energy of Spain and the European Community.

\section{Introducción}

El desarrollo de nuevas tecnologías, especialmente en los campos de la información, configuran un nuevo marco de actuación, que obliga a modificar constantemente los modelos tradicionalmente conocidos en el campo de la (c) Consejo Superior de Investigaciones Científicas Licencia Creative Commons 3.0 España (by-nc) construcción, del urbanismo, así como de las formas de difusión de las actuaciones generadas en estas áreas.

En este contexto, los participantes en el proceso (empresas, administración, corporaciones locales, etc.) deben ser capaces de introducir todas las posibilidades de las nuevas 
tendencias tecnológicas, a fin de facilitar la gestión que la redacción del planeamiento y su publicación requieren.

En la Facultad de Informática de la U.P.M. se formó, con este fin, un grupo de trabajo formado por profesores y alumnos, dentro del cual se llevó a cabo una experiencia piloto, el Proyecto S.I.C., que bajo el patrocinio del Ayuntamiento de Boadilla del Monte, aunó las Técnicas Gráficas, disciplina docente de su plan de estudios, con materias en las que su presencia es fundamental, Internet y Sistemas de Información Geográfica, GIS.

De esta manera se pudo aunar la potencia de análisis y versatilidad que ofrece el GIS, con la facilidad de manejo que ofrecen las herramientas de navegación en Internet. Así, sin tener ningún conocimiento informático previo y manejando únicamente un ratón de ordenador, es posible realizar la consulta más complicada a través del GIS, obteniendo la respuesta, de forma casi instantánea.

Lo que se pretende con este nuevo enfoque se puede sintetizar en:

Facilitar alos ciudadanos el accesoa losplanes urbanísticos, así como a cualquier tipo de información que pueda resultar de interés para el desarrollo del municipio, mediante el uso de nuevas tecnologías, Internet y GIS.

Propiciar el acercamiento de la Corporación local a los ciudadanos, agilizando los medios de comunicación entre ambos.

Proporcionar información actualizada y completa de las distintas actuaciones que lleva a cabo la Corporación

Facilitar a la Corporación municipal la gestión del municipio y la toma de decisiones.

\section{Modelo tradicional}

El Plan General de Ordenación Urbana es un documento que el Ayuntamiento redacta para estudiar la situación urbanistica del municipio y las transformaciones necesarias para afrontar su futuro.

En él se regula cómo mejorar y potenciar lo existente y dónde y de qué forma y cuándo debe crecer, respetando y protegiendo el medio urbano y rural.

Las fases de redacción de Plan General son las siguientes:

\section{AVANCE}

Es el documento de instrucciones iniciales en el que se recogen las grandes líneas de actuación.
El Ayuntamiento acuerda que se haga público a fin de recoger las propuestas y sugerencias de los ciudadanos.

\section{APROBACIÓN INICIAL}

Es el documento del Plan General en el que se pormenorizan todas las actuaciones y se incorporan las normas urbanísticas y ordenanzas. Se somete a información pública para que se puedan hacer las alegaciones oportunas.

\section{APROBACIÓN PROVISIONAL}

Es el documento que recoge las alegaciones. Se aprueba por el Ayuntamiento y se remite a la Comunidad Autóno$\mathrm{ma}$, solicitando su aprobación definitiva.

\section{APROBACIÓN DEFINITIVA}

Es el documento finalmente aprobado, cuya competencia corresponde a la Comunidad Autónoma.

\section{Proyecto S.I.C.}

Se ha llamado Sistemas de Información Ciudadana a aquellos sistemas que ofrecen, al habitante de una ciudad, información sobre los aspectos que le conciernen, en la vida diaria o relativos a sus relaciones con la Administración. Los transportes o la localización de calles, hospitales y farmacias forman parte del primer grupo. El conocimiento de Normas Urbanísticas, aplicables a una zona o a sus valoraciones catastrales, pueden ser un ejemplo de los aspectos citados en segundo lugar. Ésta es la razón de que los Sistemas de Información Ciudadana guarden una estrecha relación con los Sistemas de Información Geográfica (GIS), diferenciándose de ellos en la forma de acceder a la información. No se trata de acceder a una Base de Datos determinada de un GIS, entrando desde la estación de trabajo que almacena los datos. Se trata de acceder a ésta $u$ otras bases de datos a través de Internet, en un proceso de navegación.

Esta cuestión lleva a la distinción entre información pasiva y activa. En principio, siempre es posible presentar información fija, tanto textual como gráfica, en una página Web con algún tipo de información estructurada en una base de datos. Por otra parte, si se ha de atender a preguntas inesperadas, la respuesta tendría que elaborarse dinámicamente, es decir, deberían prepararse mediante un programa conectado a una página mediante lo que se conoce como Common Gateway Interface (CGI). Dicho programa necesitaría ciertas características de un GIS, lo que nos retrotraería a la situación anterior.

En resumen, aunque sería posible elaborar un conjunto de páginas fijas con un propósito determinado, todo parece 
indicar que la clave de la cuestión estriba en la existencia de ciertas Bases de Datos, geográficas y no geográficas, y en el acceso a las mismas a través de Internet.

\section{Aplicación al avance del Plan General de Ordenación de Boadilla del Monte}

En el Ayuntamiento de Boadilla del Monte, municipio situado a $16 \mathrm{~km}$ al S.O. de Madrid, el pasado mes de junio se aprobó, por el Pleno, someter a participación pública el Avance de Revisión del Plan General de Ordenación Urbana, redactado por Arnaiz Consultores, durante un período de tres meses, a fin de que se puedan hacer llegar las sugerencias y propuestas oportunas.

La innovación, sin precedentes en esta fase de participación pública del documento de Avance, consiste en la incorporación de la parte principal de la documentación del Avance en Internet, páginas elaboradas en el marco del Proyecto S.I.C., a fin de que el ciudadano pueda conocer el documento y enviar sus sugerencias

La implementación del Avance de dicho Plan a través de Internet posibilita el acceso de todos los ciudadanos a una serie de informaciones de utilidad legal:

. Posibilidad de conocer la calificación del suelo de cualquier punto del municipio.

Facilidad en la búsqueda mediante formularios de Dirección, Calle y Ámbito Urbanístico.

Información puntual sobre las previsiones del Ayuntamiento para una determinada zona.

Enlaces dinámicos con las fichas urbanísticas correspondientes a cada zona de actuación.

$\mathrm{El}$ proyecto consiste en un sistema de información al que se accede de manera gratuita a través de Internet, tecleando los caracteres: htpp://vauxhall.1s.fi.upm.es/boadilla, navegando con cualquier aplicación estándar, Netscape, Navigator, etc.

Para la realización del trabajo se ha contado con los siguientes medios técnicos:

. Una estación Sun Sparc-20, con acceso completo a Internet, la cual se ha utilizado como servidor de las páginas Web y del resto del software.

Netscape Enterprise Server, sistema software que facilita la instalación y gestión de un servidor Web.

- Genamap, Sistema de Información Geográfico que alberga toda la información del municipio y genera las respuestas a las consultas formulares.

(c) Consejo Superior de Investigaciones Científicas Licencia Creative Commons 3.0 España (by-nc)
La pantalla principal despliega tres apartados, que se corresponden con otras tantas áreas de información del sistema (Gráfico nº 1).

Tras la pantalla principal, se suceden los capítulos que recorreremos en este artículo, emulando el trayecto que seguirá un navegante por el Proyecto S.I.C.

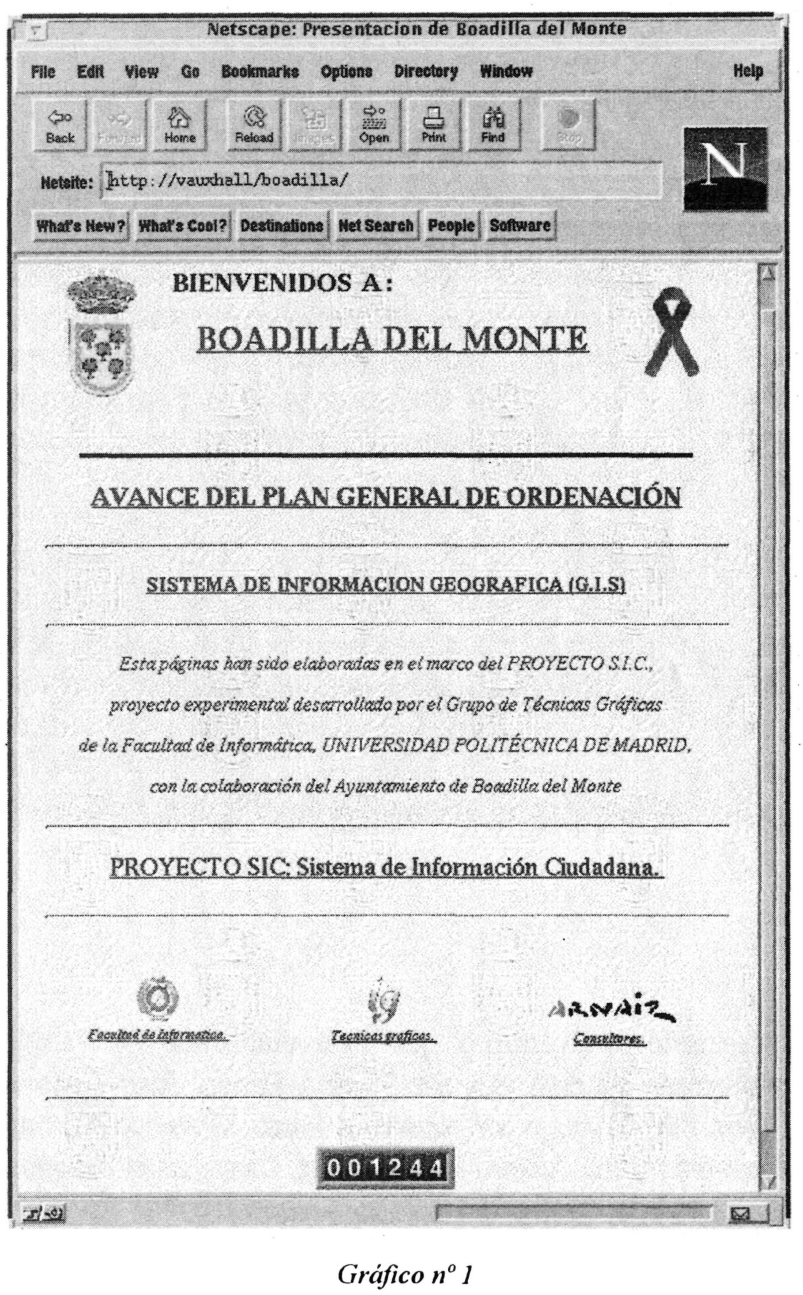

\section{1) BOADILLA DEL MONTE}

Este apartado informativo consta de tres páginas perfectamente delimitadas. En primer lugar, una página de emplazamiento del municipio (Gráfico $n^{\circ} 2$ ), desde la cual se puede acceder a dos páginas distintas, una, que recoge una síntesis de la historia del municipio (Gráfico $n^{\circ} 3$ ) y otra, que despliega los miembros de la Corporación municipal al completo.

\section{2) SISTEMA DE INFORMACIÓN CIUDADANA}

Este capítulo recoge dos páginas diferenciadas. La primera consiste en una descripción del proyecto, sus objetivos e 


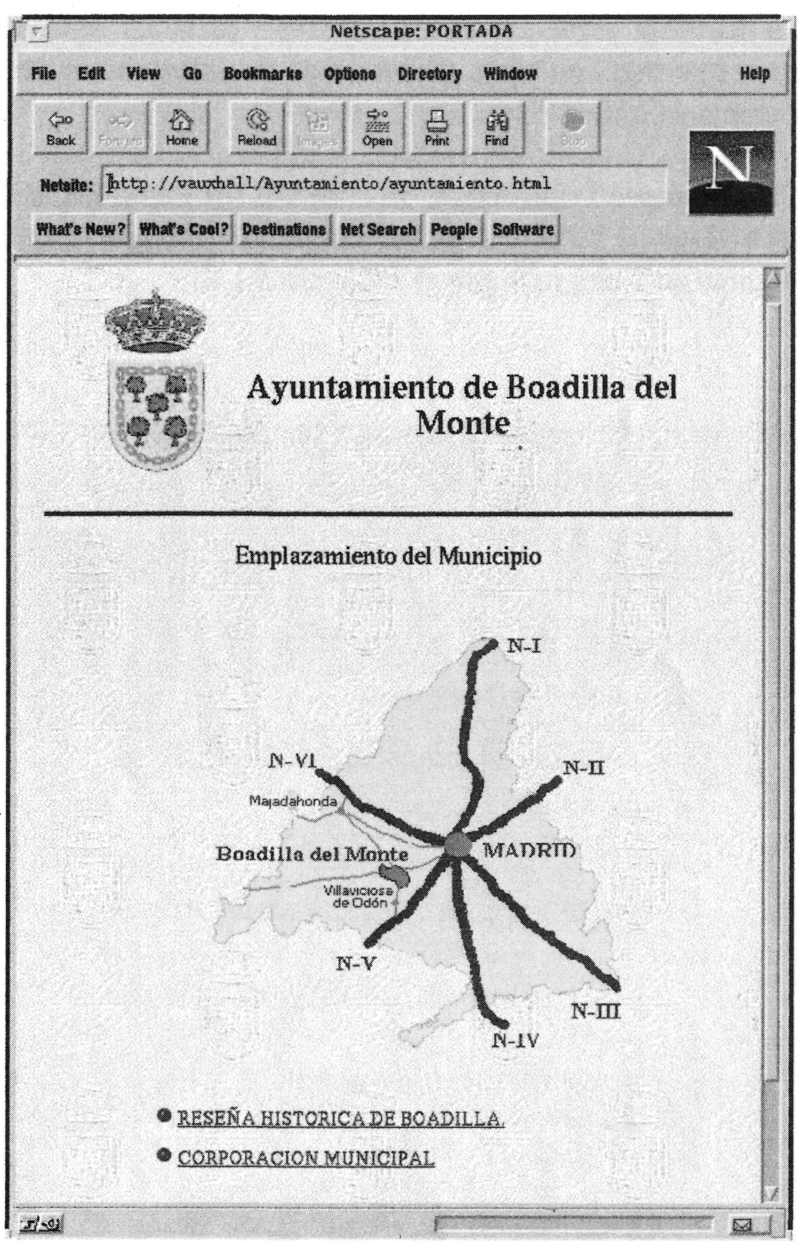

Gráfico $n^{o} 2$

integrantes del equipo que han elaborado el trabajo, profesores Marina Álvarez, Libia Pérez, José Antonio Pérez Ruy-Díaz, y los alumnos Isaac Moreno, Antonio Guevara y Juan Antonio Hernández. La segunda, contempla el grupo dentro del marco académico, las líneas de investigación que sigue, así como los trabajos y proyectos de investigación en que han participado.

\section{3) AVANCE DEL PLAN GENERAL DE ORDENACIÓN}

Este apartado constituye la esencia del sistema. La pantalla de presentación despliega ocho apartados que se corresponden (Gráfico $\mathrm{n}^{\circ} 4$ ) con distintas áreas del documento de Avance.

Por su interés destacaremos:

\section{1) Información urbanistica}

Es el corazón del sistema. En el se recoge toda la información gráfica que contempla el Avance, la cual se somete a información pública.

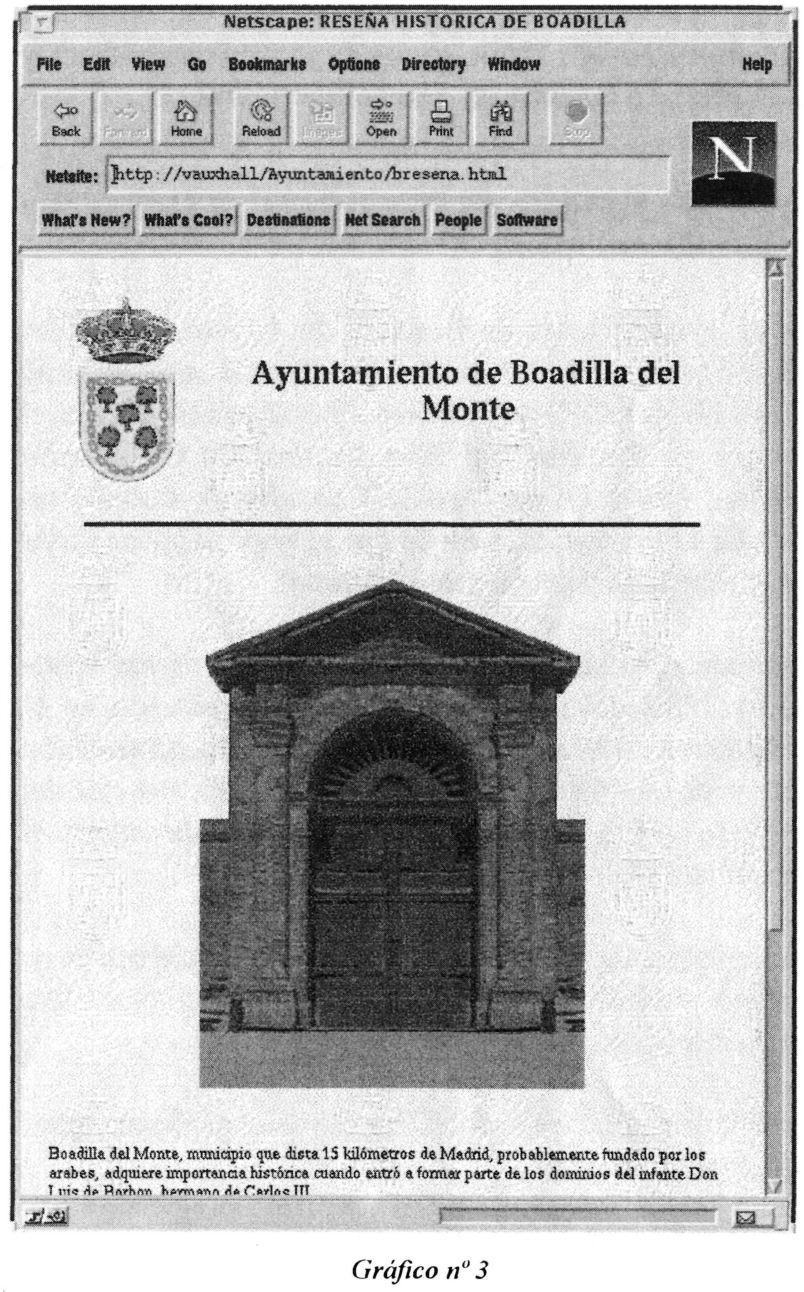

Existen dos niveles de acceso. El primero, Consulta De Fichas Urbanísticas, corresponde al acceso a un modo estático de presentación de la información: acceso pasivo. El segundo, permite conectar con un Sistema de Información Geográfica, GIS, que permite, a su vez, acceder dinámicamente a la información almacenada en una base de datos: acceso activo.

Si se utiliza el acceso pasivo (Gráfico $n^{\circ} 5$ ), empleado por primera vez en la presentación de un plan urbanístico, se recoge información acerca de los diferentes ámbitos urbanísticos en que se ha dividido el municipio. A ellos se puede acceder según la clasificación que atiende a los distintos tipos de suelo, contemplada por la vigente Normativa.

Una vez elegido el ámbito deseado, se accede a una serie de páginas que ofrecen información gráfica y las diferentes propuestas urbanísticas que le afectan, así como a una serie de fichas que recogen toda la información urbanística relativa al ámbito.

Si el usuario opta por la segunda forma, acceso activo, 
(Gráfico ${ }^{\circ} 6$ ), además de la información del documento de Avance, es posible acceder a otros datos relevantes sobre la geografia del municipio.

Este apartado supone una innovación tecnológica añadida a la que se supone el soporte del proyecto en Internet, ya que se fundamenta en la conexión de un sistema GIS a Internet, al que se pueden realizar una serie de consultas con gran sencillez.

El proyecto contempla un sistema de búsqueda de información variado, por Ámbitos, Calles y Direcciones (Gráfico $\mathrm{n}^{\circ} 7$ ). A continuación se describe brevemente el sistema de información basado en el GIS.

\section{BÚSQUEDA POR ÁMBITOS}

Al elegir esta opción accede al formulario de búsqueda por Ámbitos Urbanísticos. En dicho formulario se mostrará una lista completa de los Ámbitos de que consta el documento, así como varias opciones de visualización.

\section{BÚSQUEDA POR CALLES}

Por medio de esta opción accede al formulario de búsqueda por Calles, mediante el cual se puede localizar la práctica totalidad de las calles del municipio, admitiendo patrones aproximados de búsqueda, en caso de no conocer exactamente el nombre de la calle.

\section{BÚSQUEDA POR DIRECCIÓN}

Esta opción permite acceder al formulario de búsqueda por Dirección. Dicho formulario permite introducir direcciones postales para su localización. Las calles existentes se pueden consultar en el listado de calles.

\section{2) Información detallada de alternativas M-50}

Debido a la especial preocupación de la Corporación por el proyecto redactado por el Ministerio de Fomento para la futura M-50, ya que su diseño inicial divide al municipio, se ha introducido un capítulo en el cual se hace conexión a las páginas Web, en las que se pueden consultar los estudios informativos aprobados inicialmente por la Dirección General de Carreteras, referentes a los trazados futuros de la citada vía. Estas páginas son el resultado de una exposición realizada en el citado Ministerio.

\section{3) Sugerencias y comunicaciones}

El Proyecto S.I.C. es un sistema abierto al diálogo. Siguiendo el objetivo de facilitar el acercamiento entre la Corporación y los ciudadanos, cualquier usuario interesado puede consultar o proponer sugerencias sobre el Plan, rellenando un formulario que aparece en la página $\left(\right.$ Gráfico $\left.n^{\circ} 8\right)$, el cual, con el fin de ser atendido, es mandado a una dirección de correo electrónico habilitada al efecto.

\section{Resultados}

La aplicación del S.I.C., hasta la fecha, está teniendo un gran éxito, más de 700 accesos desde el día de la inauguración -a primeros de julio- debido a que facilita, por una parte,. el acceso a los ciudadanos a cualquier tipo de información de interés para el desarrollo del municipio y, a su vez, facilita el acercamiento de la Corporación a los ciudadanos, agilizando los medios de comunicación entre ambos.

En cuanto al perfil de los visitantes, tal y como cabía esperar, el acceso ha sido realizado, en su mayoría, por usuarios españoles, tanto particulares como de Instituciones Públicas (C.S.I.C., Gobierno de Aragón, Universidades) y Empresas(Telefónica, CASA, Oracle, Compaqy Ericson). Asimismo se puede destacar la conexión de navegantes de varios países de Europa (Gran Bretaña, Suecia) y de América (EE.UU, Brasil, Chile, etc.).

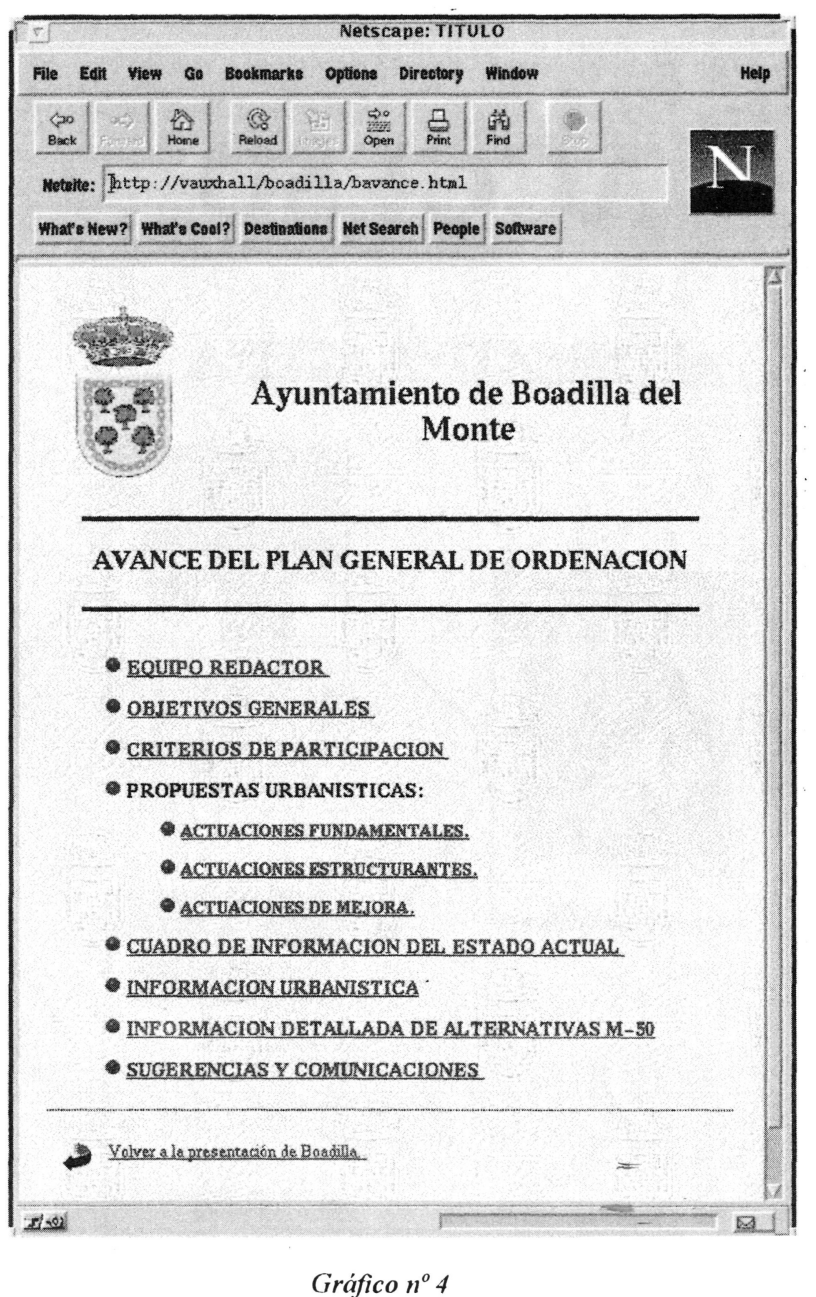




\section{ACCESO}

\section{ALTERNATIVA PASIVA:}

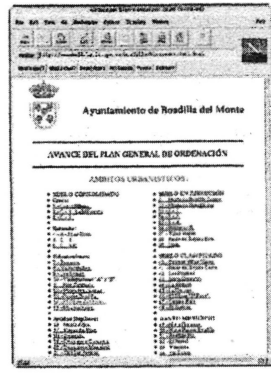

INDICE

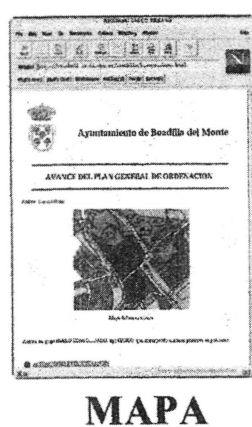

AMBITO

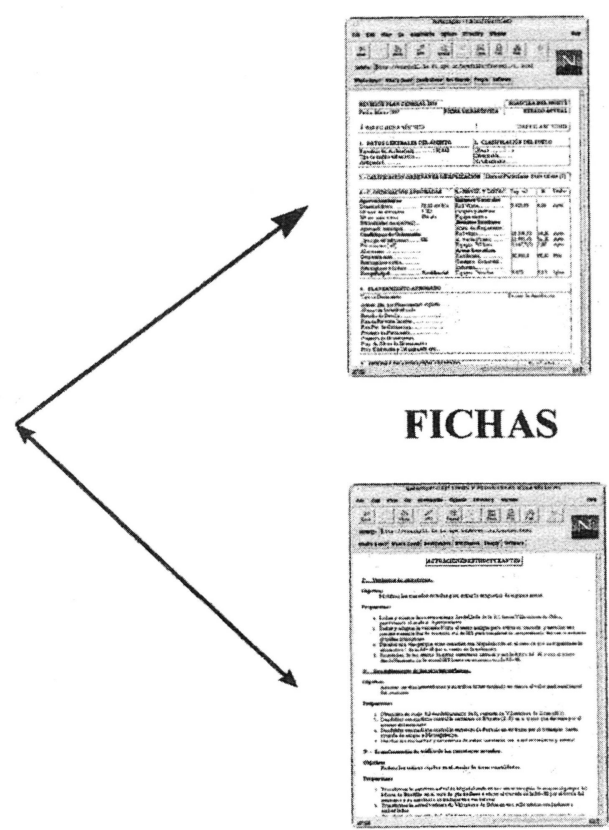

MEMORIA

Gráfico $n^{\circ} 5$

\section{ACCESO}

\section{ALTERNATIVA ACTIVA}
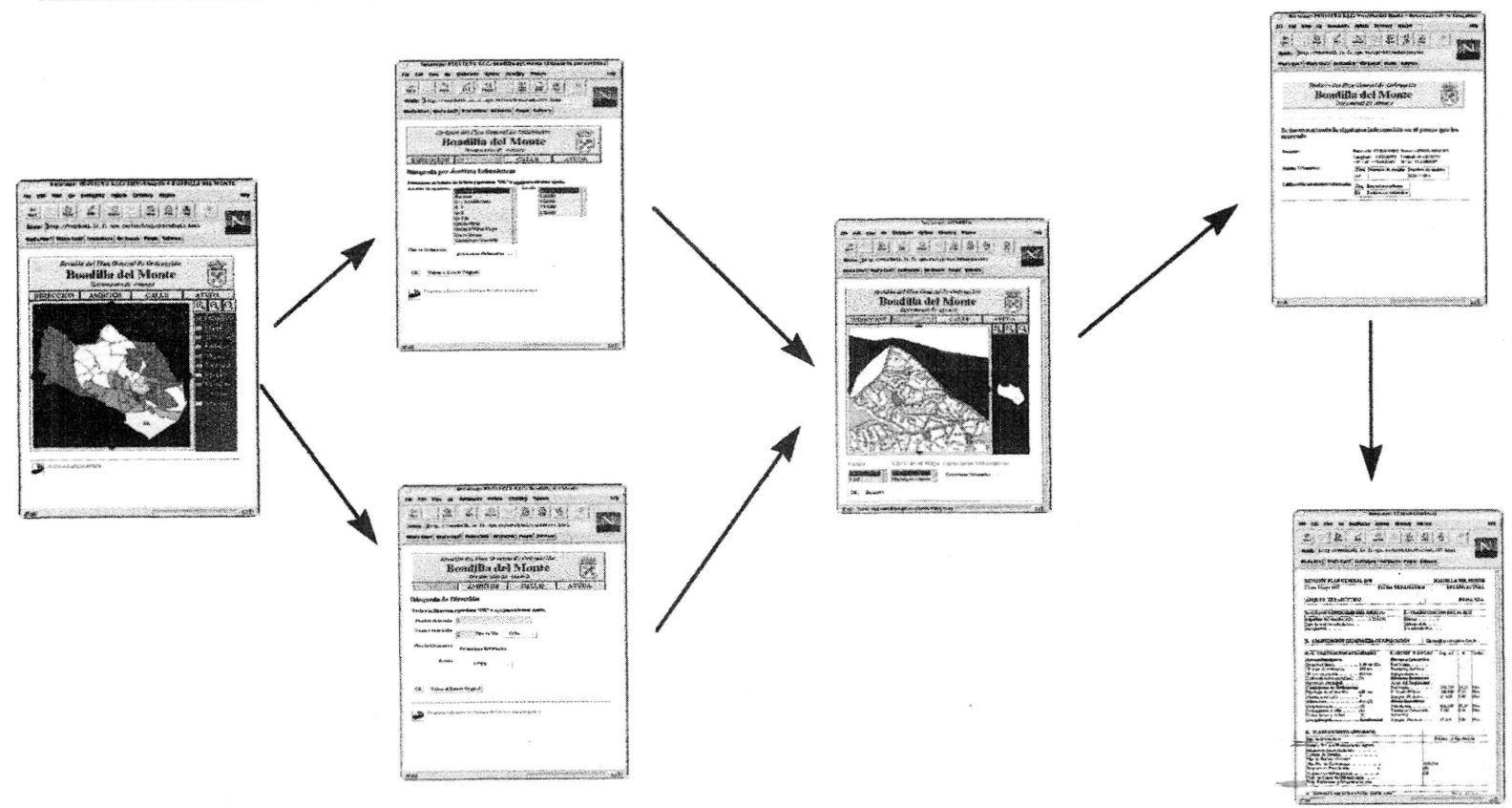

Ciráfico $n^{\circ} 6$ 


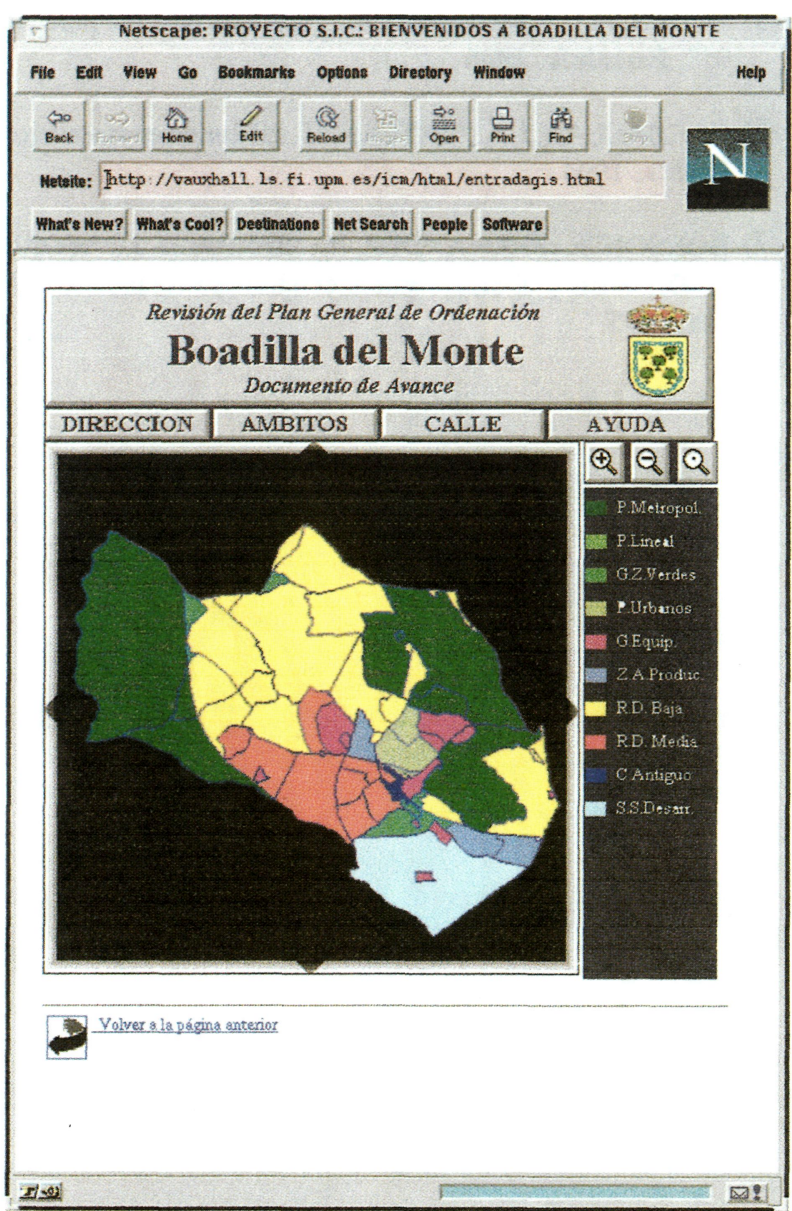

Gráfico $n^{\circ} 7$

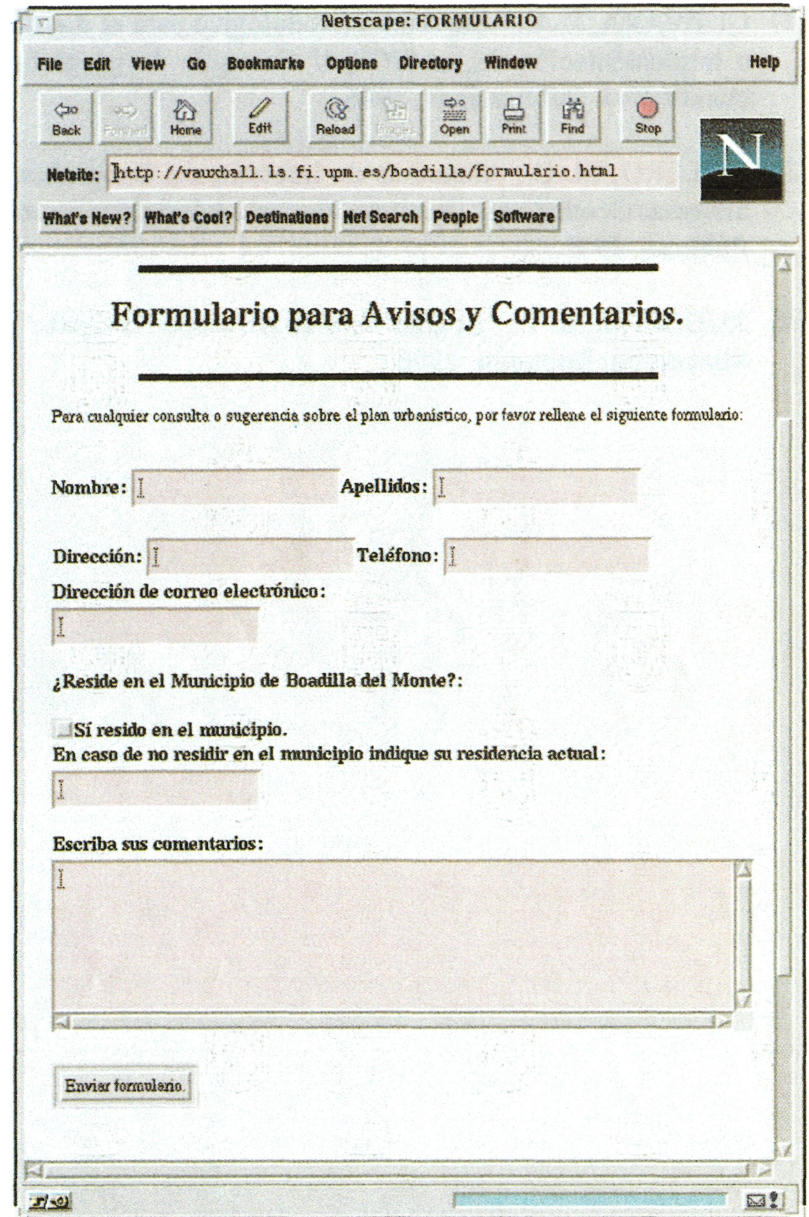

Gráfico $n^{\circ} 8$
El siguiente paso, respecto a este tipo de aplicaciones de consulta pública, se dirige a la implementación de un sistema similar, pero dirigido al uso interno de una Corporación local mediante una Intranet. De este modo, puesto que se puede asociar cualquier tipo de información que se desee, por ejemplo, los planos completos de una vivienda o los planes de urbanismo de una zona concreta, se podrían agilizar ciertas gestiones municipales (licencias, etc.).

\section{Conclusiones}

- En este artículo se ha analizado y propuesto un procedimiento, Proyecto S.I.C., pionero en el sector, en el cual se aúnan dos tecnologías avanzadas, Internet y GIS en la difusión del Planeamiento. Este procedimiento puede ser aplicable, a su vez, a diferentes áreas relacionadas con el proceso constructivo.

- El Proyecto S.I.C. proporciona un conjunto de elementos de información y comunicación, fundamentales para la difusión y participación pública del Planeamiento urbanistico.

(c) Consejo Superior de Investigaciones Científicas Licencia Creative Commons 3.0 España (by-nc)
- La creciente aplicación de las Intranets, unido a la facilidad con que se pueden realizar las consultas al sistema GIS (basta navegar con cualquier aplicación estándar, Netscape, Navigator, etc.), hacen que este nuevo soporte se convierta en una herramienta imprescindible en la gestión municipal.

- La filosofía del sistema distribuido que implica Internet, hace que el coste de implementación sea despreciable frente a los sistemas GIS tradicionales.

- La utilización de interfaces en forma de páginas Web.(formularios), confieren gran flexibilidad al sistema, pudiendo generarse aplicaciones a medida.

- El uso de navegadores estándar implica coste cero en cuanto al aprendizaje de los usuarios, ya sean ciudadanos o funcionarios municipales.

\section{BIBLIOGRAFÍA}

(1) MOLDES, J.; "Tecnología de los Sistemas de Información Geográfica. (SIG)”, Ra-Ma, 1995. 
(2) GUEVARA, J. A.; "Esquema metodológico para el diseño e implementación de un SIG", V Coloquio de geografía cuantitativa. Publications, 1996.

(3) MELERO, M. C.; "Sistemas de Información Geográfica. Sistemas Geomáticos", Instituto Vasco de Administraciones Públicas, 1995

(4) RUDOLPH, M. T.; "El gran libro de Netscape Navigator", Marcombo, Boixerau, 1996
(5) JAMES, PH, WEINGARTEN,; "Guía de Internet para Win 95", Paraninfo, 1996

(6) CARBALLAR, J. A.; "INTERNET, el Mundo en sus manos", Ra-Ma, 1994

(7) BONSON, E.; FERNÁNDEZ, R.; SIERRA, G. J.; "WORLD WIDE WEB y el ciberespacio económico-financiero", RaMa, 1995.

\title{
Publicación del Instituto Eduardo Torroja - CSIC
}

\author{
Número monográfico de INFORMES
}

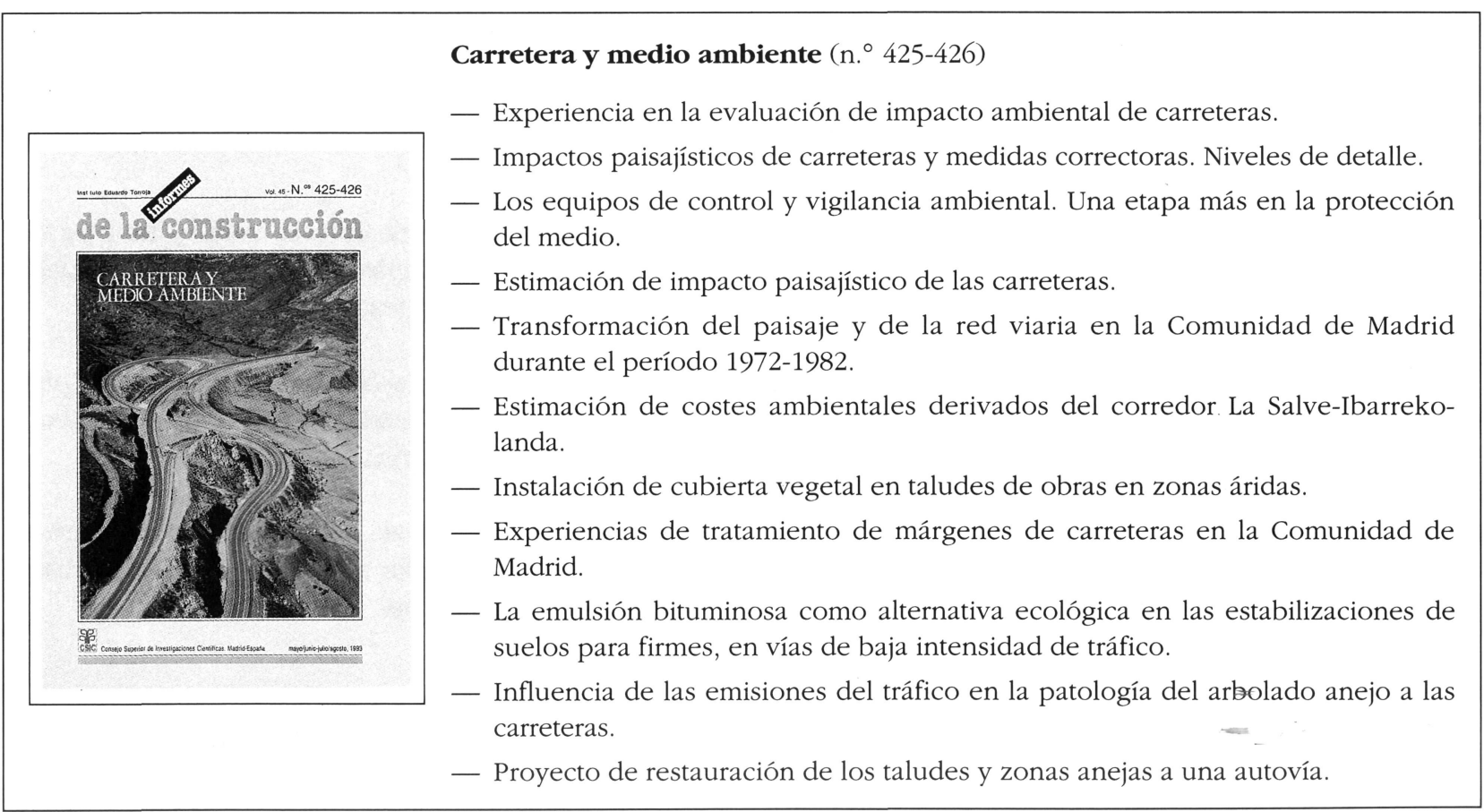

